\title{
The importance of being 'in charge'
}

Lila Schope, the Attending Veterinarian $(\mathrm{AV})$ at Great Eastern University, was also the Director of the animal care program. As such, she was always looking for ways to bring in new revenue to help keep down the per diem animal care charges. For over three years she had been receiving requests from local startup companies to house mice and guinea pigs. Because animal housing space was available at Great Eastern, she began the process of entering into contractual agreements for animal housing. The companies would provide any technical personnel needed for research procedures. After all legal and National Institutes of Health requirements were in place, the university's attorneys and the Vice President for Research approved the contracts.

Great Eastern had a policy that only a university employee could be a Principal
Investigator (PI) on an IACUC protocol, so Schope listed herself as the PI on each individual research protocol that involved work with the contracted companies. Schope felt that this was appropriate because as the $\mathrm{AV}$ she had general oversight responsibility for all the animal research performed on campus. The IACUC agreed and it approved her protocols. At first there was no problem, but after two years the IACUC, led by a new Chairperson, told her that she could no longer be PI unless she was "intimately involved with the research." Of course, Schope was upset because the Committee was changing its decision in the middle of work on 12 contract protocols from four different companies. She was trying to help the university and she had no desire to be any more involved in the research than she originally stated to the IACUC. The IACUC said that it was simply complying with federal regulations about the responsibilities of a PI. Schope said that the only regulation that even vaguely touched upon the current issue was the definition of a PI in the Animal Welfare Act regulations (AWRs). Nevertheless, she contended that there was no appropriate regulatory guidance that the IACUC could use to justify replacing her as PI. She also wanted to know what would happen to the research if her PI status was revoked. The Vice President for Research sided with the IACUC.

Was Shope correct to assert that there is no applicable regulatory guidance for either her or the IACUC? Would it be better for everybody if Shope just threw in the towel and discontinued the outside companies' animal housing and research activities? What do you recommend that Great Eastern and Schope do to resolve this conflict?

\section{RESPONSE}

\section{A compromise for co-PIs}

\section{Harry Rozmiarek, DVM, PhD, ACLAM \& Glenn Rall, PhD}

It is important to note that the policy on which this issue is based is a universityestablished regulation at Great Eastern, not a specific federal mandate. While it is the responsibility of the research facility to ensure that all are qualified to perform their duties and that personnel conducting procedures on the research animals are appropriately qualified and trained in those procedures, there is no federal requirement that only a university employee can be a PI on an approved IACUC protocol. Thus, the former arrangement was compliant with federal standards as long as Schope is familiar with the research and qualified to function as the PI. However, because the PI is responsible for all aspects of animal care and use described in the protocol, it would be best for that person to be intimately associated with the experiments described in the protocol. In this particular situation, this is clearly not Schope. The key issue, therefore, is whether a balance can be achieved between appropriate animal oversight and compliance with university policies.

Another important point to consider is that IACUC policies need to be consistent in order to be credible. Reversal of longstanding policies simply because a new chairman deems them no longer acceptable sends a confusing message to the investigators and may prevent valuable research from moving forward. Nevertheless, the new IACUC Chair does have a valid concern in that the PI may not be appropriately familiar with the proposed research, and is listed as PI mainly to comply with a
Great Eastern-established policy. One possible remedy that would enable the work to move forward while allowing for someone more familiar with the research to be responsible for the day-to-day monitoring and care of the animals is for the Great Eastern IACUC to consider the idea of co-PIs. Schope could serve as the university representative, along with a non-Great Eastern individual from the contracting company who is actually doing the work. Both would share in the responsibilities of monitoring animal welfare and, in this way, provide adequate surveillance of the animals, while still adhering to the Great Eastern policy.

Rozmiarek is Professor Emeritus, University of Pennsylvania, Philadelphia, PA, and Director Laboratory Animal Facility, Fox Chase Cancer Center, Philadelphia, PA. Rall is IACUC Chairman and Associate Professor, Fox Chase Cancer Center and Adjunct Professor, University of Pennsylvania. 